${ }^{1}$ National Renewable Energy Laboratory, Golden, CO, USA

${ }^{2}$ Arable Labs, Inc., Princeton, NJ, 08542, USA. ${ }^{3}$ Solar Consulting Services, Colebrook, NH, USA

\begin{abstract}
meter sensing and measurement devices enable cost-effective monitoring of the functional, operationa reliability, efficiency, and resiliency of the electric grid.

The National Renewable Research Laboratory (NREL) Solar Radiation Research Laboratory (SRRL), in collaboration with Arable Labs, Inc., deployed Arable Lab's Mark multiparameter sensor system.

The device measures the downwelling and upwelling shortwave solar resource and longwave radiation, humidity, air

temperature, and ground temperature.

The system is also equipped with six downward- and upwardfacing narrowband spectrometer channels that measure spectra radiation and surface spectral reflectance.

- This study describes the shortwave calibration, characterization and validation of measurement accuracy of this instrument by comparison with existing instruments that are part of NRELSRRL's Baseline Measurement System.
\end{abstract}

Method

\section{Results \\ A. Shortwave Data Analysis}

To correct for directional dependency, a correction was applied as a function of solar azimuth (the bias line in Fig. 2.), as follows: $E_{\text {cor }}=E_{\text {raw }}-\sum_{0}^{6} a_{i} A^{i}$

where $E_{\text {cor }}$ is the corrected irradiance for the unit under test (UUT), $E_{\text {raw }}$ is the uncorrected (raw) irradiance, $A^{i}$ is the solar azimuth, and $a_{i}$ are numerical coefficients obtained by least-squares fitting.
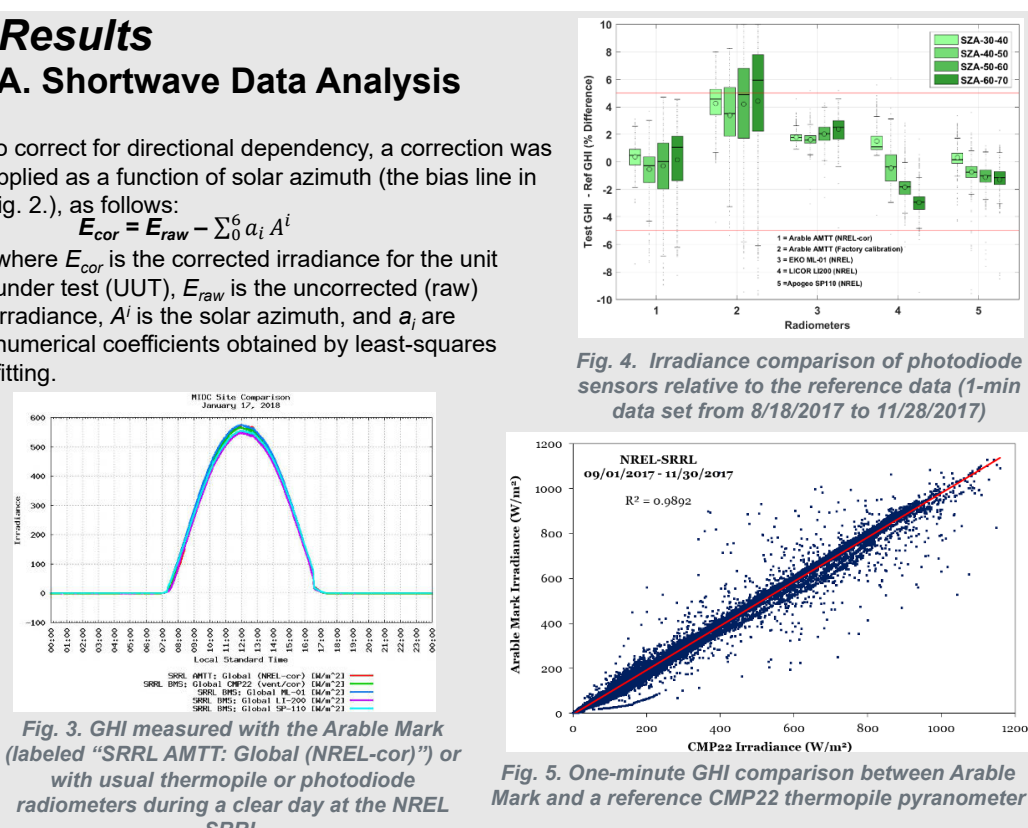
$+2 \%$ bias compared to $2 \%-8 \%$ when using only the factory methodology. Further, the NREL-corrected UUT shows a comparable or better result, with average bias of less than $\pm 1 \%$ compared to the three
conventional photodiod sensors.

A long-term analysis included an all-sky comparison at 1-minute temporal resolution during a 3-month period (8/18/2017 to $11 / 28 / 2017)$ The results show good agreement with an Rsquare value of $\sim 0.99$ compared with the reference CMP22

Fig. 5. One-minute GHI comparison between Arable instrument.

Mark and a reference CMP22 thermopile pyranometer

\title{
Conclusions and Future Work
}

The comparison of the Arable Mark device demonstrated good agreement with the existing photodiode pyranometers, such as the LI-200 sensor from LICOR (with an average bias of less than $\pm 1 \%$ ).

- The calibration and characterization methodologies employed by NREL result in $\pm 2 \%$ bias compared to the reference GHI data obtained with a thermopile pyranometer during a 3-month period.

- The spectral capabilities of the Mark instrument were found satisfying compared to a reference spectroradiometer, at least under clear-sky conditions.

- Future work will characterize shortwave and spectral data from the Mark device on fixed-tilt, one-axis, and dual-axis tracking in addition to further characterize longwave, temperature, and humidity data.

\section{References}

\section{B. Spectral Data Analysis}
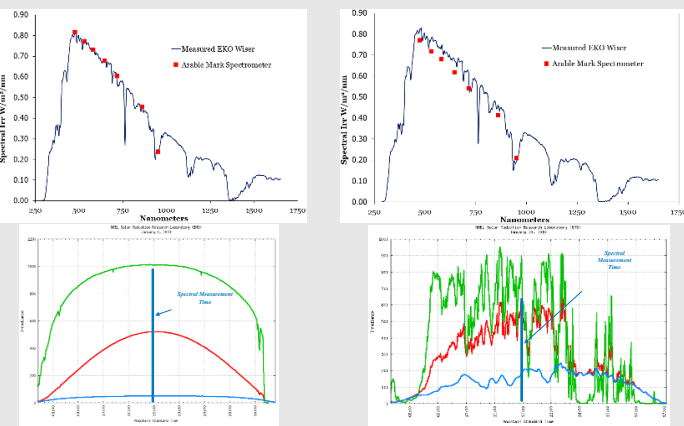
@ 12:00 LST; (bottom) broadband direct (green), global (red), and diffuse (blue) same but for partly cloudy skies for 01/20/2018 @ 12:00 LST (right) A similar least-squares fitting function as the shortwave is used to correct the error. After correction, the Mark and WISER instruments show good agreement under clear-sky conditions (Fig. 6, left).

- Under partly cloudy skies, the difference is significant. This can be partly attributed to the difference in scan rate because the WISER scans every 5 minutes, whereas the spectrometer scans each minute. Sky conditions and atmospheric transmittance can change rapidly under cloudy situations, hence triggering rapid variations in the solar spectrum (Fig. 6, right).

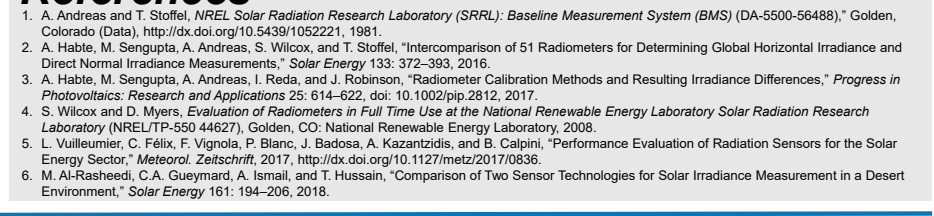

